\title{
The Photopolymer Science and Technology Award
}

The Photopolymer Science and Technology Award No. 160200, the Best Paper Award 2016, was presented to Hiroki Takano ${ }^{a}$, Lei Wang ${ }^{a}$, Yuki Tanaka ${ }^{\mathrm{a}}$, Rina Maeda ${ }^{\mathrm{a}}$, Naoko Kihara ${ }^{\mathrm{b}}$, Yuriko Seino ${ }^{\mathrm{b}}$, Hironobu Sato $^{\mathrm{b}}$, Yoshiaki Kawamonzen ${ }^{\mathrm{b}}$, Ken Miyagi ${ }^{\mathrm{b}}$, Shinya Minegishi ${ }^{\mathrm{b}}$, Tsukasa Azuma ${ }^{\mathrm{b}}$, Christopher K. Ober ${ }^{\mathrm{c}}$, and Teruaki Hayakawa ${ }^{\mathrm{a}}$ ( ${ }^{\mathrm{a} D e p a r t m e n t}$ of Organic and Polymeric Materials, Tokyo Institute of Technology, ${ }^{b} E U V L$ Infrastructure Development Center, Inc, ${ }^{\mathrm{c}}$ Materials Science \& Engineering, Cornell University) for their outstanding contribution published in Journal of Photopolymer Science and Technology, 28, (2015) 649-652, entitled "Vertical Oriented Lamellar Formation of Fluorine- and Silicon-containing Block Copolymers without Neutral Layers".

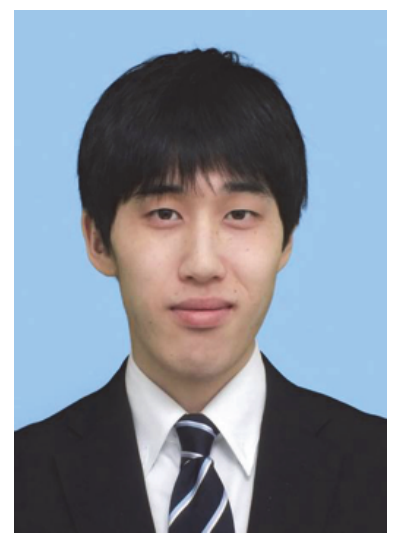

Hiroki Takano

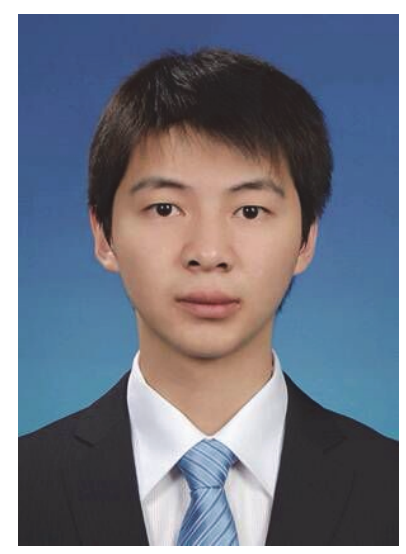

Lei Wang

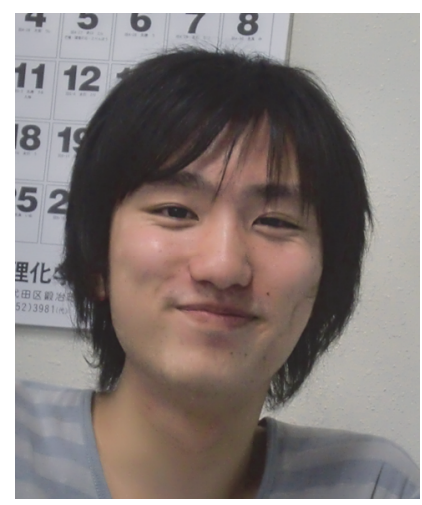

Yuki Tanaka
Hiroki Takano is a second year of graduate student in the Department of Organic and Polymeric Materials at the Tokyo Institute of Technology. He focuses on the design and synthesis of various methacrylate polymers for block copolymer lithography.

Lei Wang studied organic and polymeric materials at the Beijing University of Chemical Technology and Tokyo Institute of Technology, received a M.S. and a Dr. of Eng. in 2011 and 2014, respectively. After graduation he worked as a postdoctoral researcher at Tokyo Institute of Technology, where he coorperated with the researchers from EUVL Infrastructure Development Center (EIDEC), Inc. His research focuses on the design and synthesis of novel block copolymers for sub-10 nm lithographic materials and directed selfassembly. In 2016, he will be a postdoctoral fellow under Dr. Redouane Borsali, Dr. Patrice Rannou and Prof. Olli Ikkala's guide in CERMAV-CNRS, France.

Yuki Tanaka received a master degree in 2015 from the Department of Organic and Polymeric Materials at the Tokyo Institute of Technology. His research focused on the synthesis and bulk \& thin film morphologies of fluorine-containing block copolymers. 


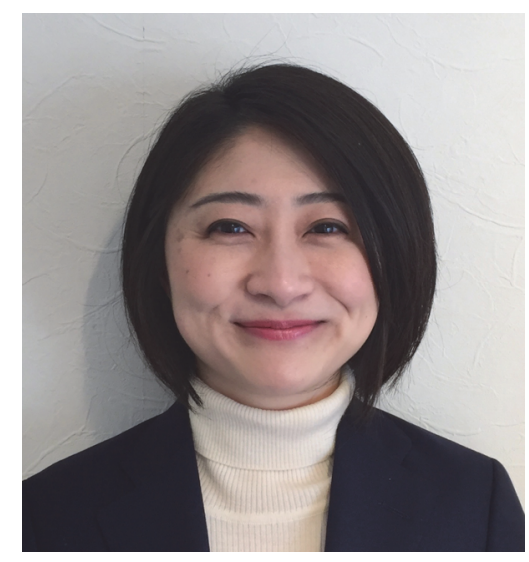

Rina Maeda

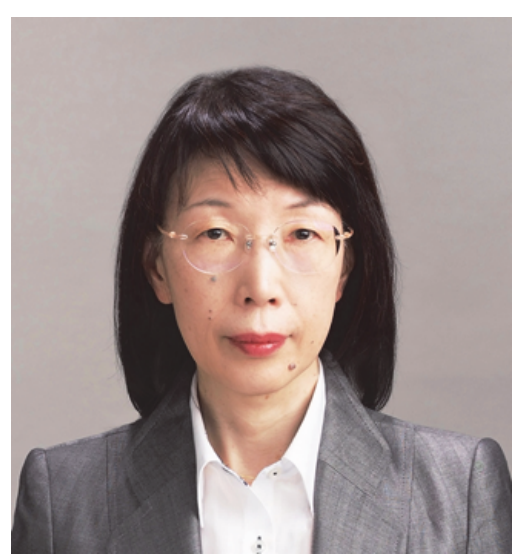

Naoko Kihara

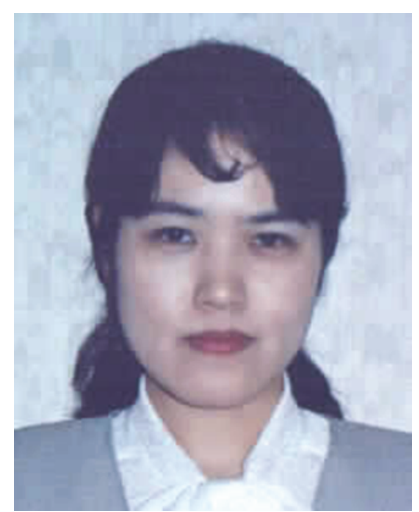

Yuriko Seino

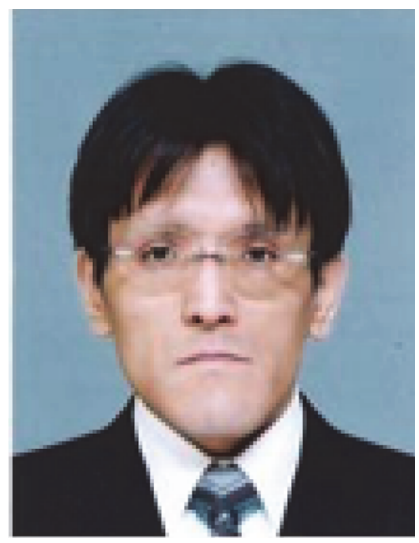

Hironobu Sato
Rina Maeda received the B.Eng. degree in 2006, the M.Eng. degree in 2008, and Ph.D. in 2013 under the supervision of Prof. Teruaki Hayakawa at Tokyo Institute of Technology, Japan, where she studied synthetic polymer chemistry and self-assembling material such as liquid crystalline polymers and block copolymers (BCPs). During her Ph.D. study, she visited Ober group at Cornell University, U.S. Her Ph.D. thesis focused on the role of partially fluorinated polymer on the BCP assembly and application for the nanolithography. Rina joined Weibel group at University of Wisconsin-Madison, U.S. as a postdoctoral scholar and investigated the effect of liquid crystalline materials on the swimming and migrating behavior of microorganisms. Rina is an Assistant Professor in the Ito laboratory at the University of Tokyo. Her current research focuses on the synthesis of polyrotaxane (necklace-shaped macromolecule) with dynamic self-assembling structures and physical properties of the soft elastic polymer films.

Naoko Kihara received her M.S. degree in photochemistry from Ochanomizu University. She joined Toshiba Corporation in 1983 and has been working on organic materials for fabrication of electric devices. Since 2013, she has been working for DSA development program in EUVL Infrastructure Development Center, Inc. Currently, she is in DSA Research Department for DSA process development.

Yuriko Seino received her M.A. degree from Waseda University, Japan in 1995. She had worked at Center for Semiconductor Research \& Development, Toshiba Corporation, where she had engaged in lithography process technology development. Since 2013, she has been working for DSA development program in EUVL Infrastructure Development Center, Inc.

Hironobu Sato received his Ph.D. degree from Waseda University. In 2011 he joined Toshiba Corporation. Since 2013, he has been working for EUVL Infrastructure Development Center, Inc. His current research interest is DSA lithography for semiconductor application. 


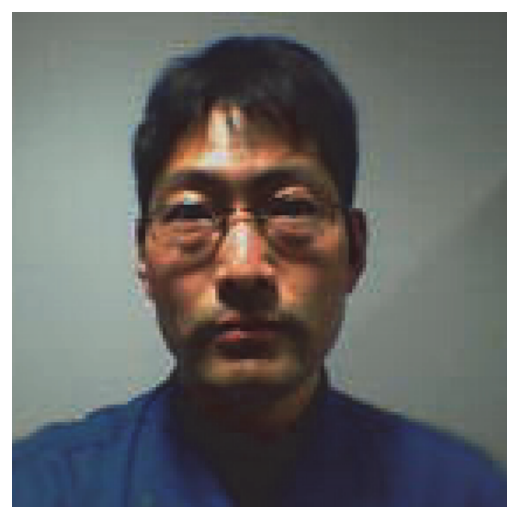

Ken Miyagi

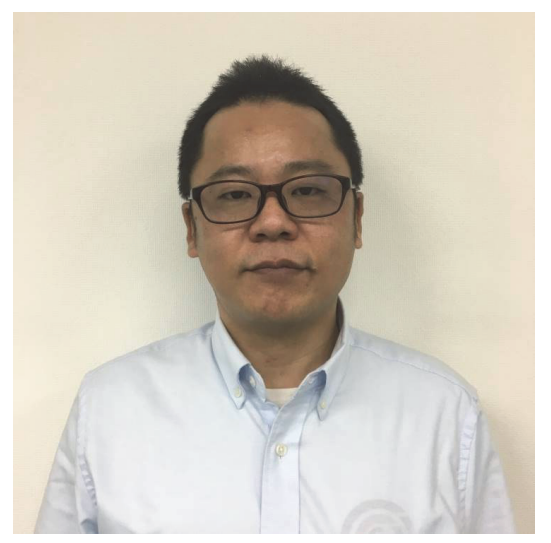

Shinya Minegishi

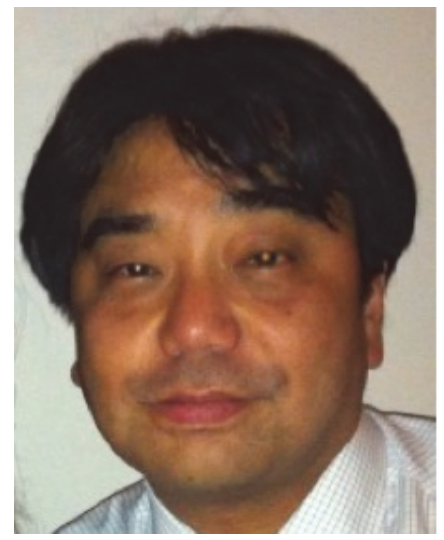

Tsukasa Azuma

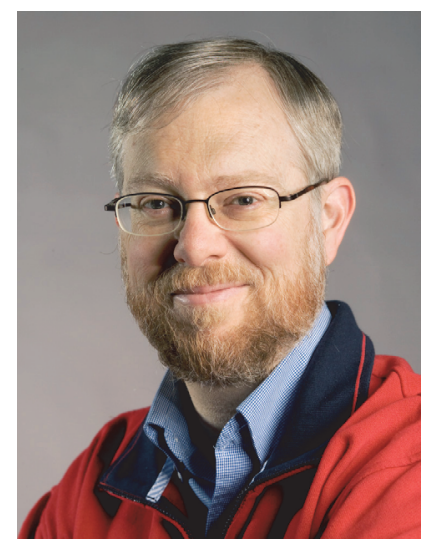

Christopher K. Ober
Ken Miyagi received B.E. and M.E. Degrees from Tokyo Institute of Technology in 1992 and 1994, respectively. He joined Tokyo Ohka Kogyo Co., Ltd. where he has engaged in the development of directed self-assembly (DSA) material. He had joined EUVL Infrastructure Development Center (EIDEC) from 2013 to 2016.

Shinya Minegishi received a bachelor (1999) degree in chemistry and a master (2001) degree in chemistry from Kyoto University Japan and Ph.D. (2004) degree in chemistry from Ludwig-MaximiliansUniversität, München, Germany. He worked as postdoctoral fellow at Kyoto University in 2004-2006. Since 2006, he has been working for the development of semiconductor materials at JSR Corporation. He is currently on temporary assignment as a researcher in EUVL Infrastructure Development Center, Inc. for the development of EUV and DSA materials and processes.

Tsukasa Azuma received B.S., M.S., and Ph.D. Degrees from Tohoku University. He joined Toshiba Corporation in 1989, where he has been engaged in advanced lithography process development for semiconductor device manufacturing. His research has focused on the advanced lithography process development in electron and photon beam technologies, nanoimprint, and directed self-assembly (DSA). He was the general manager of DSA research department, EUVL Infrastructure Development Center, Inc. (EIDEC) from April 2013 to March 2016.

Christopher K. Ober is the Francis Bard Professor of Materials Engineering, Materials Science and Engineering, Cornell University. He received B.Sc. (1978) degree in chemistry from the University of Waterloo, Canada, M.S. (1980) and Ph.D. (1982) degrees in chemistry from the Department of Polymer Science \& Engineering, University of Massachusetts, Amherst, joined the Department of Materials Science \& Engineering, Cornell University in 1986, and was promoted a full professor in 2001. He has developed new $F_{2}$ and ArF resists, block copolymer resists, and nanoparticle resists with excellent resolution and extremely high sensitivity. He was the President, IUPAC polymer division (2008-2011), and was named a Fellow of the American Chemical Society in 2009 and a Fellow of the American Association for the Advancement of Science in 2014. He has won numerous accolades, including the Outstanding Achievement Award 2015 in photopolymer science and technology. 


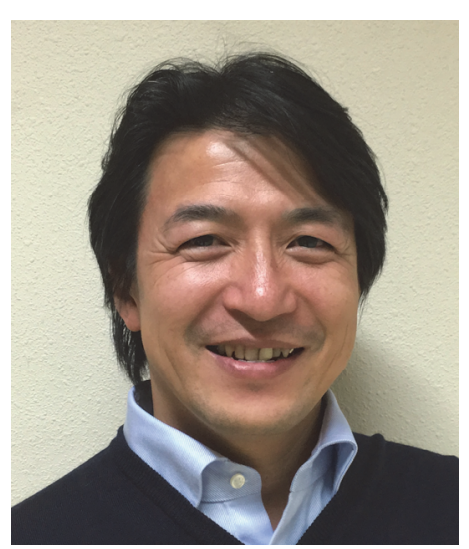

Teruaki Hayakawa
Teruaki Hayakawa is an Associate Professor in the Department of Organic and Polymeric Materials of Tokyo Institute of Technology. He received B.Sc. (1995), M.S. (1997) and Ph.D. (2000) degrees from Yamagata University with Prof. Mitsuru Ueda for research in the fields of polymer materials science and engineering. During 19971998 he was a visiting student with Prof. Christopher K. Ober at Cornell University. In 2000 he joined the National Institute of Advanced Industrial Science and Technology (AIST) as a researcher and moved to Tokyo Institute of Technology as an assistant professor in 2003, and was promoted the associate professor in 2009. He has developed block copolymer resists, high thermal conducting epoxy materials, wholly aromatic condensation polymers. He was a chairperson of the research group on Polymers for Microelectronics and Photonics, the Society of Polymer Science, Japan (SPSJ) in 20122014. He has received the Award of Encouragement of Research in Polymer Science National Science, the Society of Polymer Science, Japan (SPSJ) 2005, the SPSJ Award for the Outstanding Paper in Polymer Journal 2006 sponsored by ZEON, the SPSJ Hitachi Chemical Award 2010, and the SPSJ Wiley Award 2015.
To keep up with the demands in the microelectronics industry for smaller devices with increased pattern densities, the ability to fabricate nanopatterns in the sub-10 $\mathrm{nm}$ scale beyond conventional optical/EUV lithography is vital $[1,2]$. The self-assembled nanostructures of block copolymers (BCPs) hold the key for the development of next-generation nanolithography technologies, and particularly vertically oriented lamellar structures are crucial for the fabrication of high resolution line and space $(\mathrm{L} / \mathrm{S})$ patterns in microelectronic devices [3].

BCP materials are essential for the fabrication of thin films with vertically oriented lamellar structures with sub-10 nm feature sizes. To realize vertically oriented lamellar structures by BCP materials, controlling the balance between the surface segregation and the thermodynamic incompatibility of the blocks is important. Strongly segregating BCPs tend to avoid forming vertically oriented lamellae as a result of the large difference in the surface free energies between the blocks, whereas highly incompatible blocks are necessary to achieve smaller feature sizes. In general, there is a trade-off between increased block incompatibility and an increased tendency for the formation of vertically oriented lamellae.
Prof. Hayakawa, Dr. Maeda, Dr. Azuma, Prof. Ober and their groups proposed a novel class of fluorine- and silicon-containing BCPs as a solution to the above trade-off problems.

Based on earlier work done by the group $[4,5]$, in this paper, a series of poly(methacrylate polyhedral oligomeric silesequioxane)-blockpoly(trifluoroethyl methacrylate) (PMAPOSS- $b$ PTFEMA) BCPs with a silicon-containing PMAPOSS block and a fluorine-containing PTFEMA block $\left(\gamma_{\text {PTFEMA }}=25.1 \mathrm{~mJ} \mathrm{~m}^{-2}\right)$ were developed for the fabrication of vertically oriented lamellar structures without the use of a special neutral layer.

Two samples of PMAPOSS- $b$-PTFEMA were successfully obtained via anionic polymerization and the purification of the resulting products with recycling preparative size exclusion chromatography. The BCPs used in this study had molecular weights of $32,000 \mathrm{~g} \mathrm{~mol}^{-1}$ and 22,000 g $\mathrm{mol}^{-1}$, with narrow polydispersity indices of 1.06 and 1.07, respectively. Subsequently, morphological characterization of the bulk sample was carried out by preparing samples from solvent evaporation in chloroform and analyzed with transmission electron microscopy (TEM) and small angle X-ray scattering (SAXS). The TEM images obtained clearly showed a line-like lamellar 
morphology and the peaks of the SAXS profile obtained were characteristic to lamellae.

Further studies were conducted in the thin film via atomic force microscopy (AFM). The BCP dissolved in chloroform was spin-casted onto silicon wafers to obtain a thin film with a thickness of approximately $30 \mathrm{~nm}$. After optimizing the thermal annealing conditions to $150{ }^{\circ} \mathrm{C}$ for $1 \mathrm{~min}$ under air ambient conditions, AFM analyses were conducted that showed lines with a contrast between the PMAPOSS and PTFEMA domains. This is considered to be due the similar surface free energy between the two blocks. Oxygen plasma reactive ion etching was carried out on the annealed thin films and a trench line pattern of line width ca. $10 \mathrm{~nm}$ was obtained.

Interestingly, the vertically oriented lamellae could be obtained on various substrates such as silicon wafers, glass plates and plastics without the use of a special neutral layer. The relatively short annealing time of one minute in air ambient conditions is favorable for the mass production of nano-patterns. Therefore, PMAPOSS- $b$-PTFEMA, with its characteristic sub-10 nm scale selfassembly and good etching contrast, can be one of the new candidates for next-generation sub-10 nm BCP nanolithography.
As described above, the authors have demonstrated comprehensive fundamental research on a methodology for preparing BCPs for selfassembled nanostructures without special neutral layers. These interesting research results were presented at the Annual Conference of Photopolymer Science and Technology in 2015 and the paper was published in the Journal of photopolymer Science and Technology. This paper contributes to the understanding of the fundamental aspects to form self-assembled nanostructures.

\section{References}

1. J. Bang, U. Jeong, D. Y. Ryu, T. P. Russell, and C. J. Hawker, Adv. Mater., 21 (2009) 4769.

2. J. Y. Cheng, C. A. Ross, H. I. Smith, and E. L. Thomas, Adv. Mater. 18 (2006) 2505.

3. M. P. Stoykovich, and P. F. Nealey, Mater. Today, 9 (2006) 20.

4. T. Hirai, M. Leolukman, S. Jin, R. Goseki, Y. Ishida, M-A. Kakimoto, T. Hayakawa, M. Ree, P. Gopalan, Macromolecules, 42 (2009) 8835.

5. R. Maeda, T. Hayakawa, C. K. Ober, Chem. Mater., 24 (2012) 1454.

Seiji Nagahara, Tokyo Electron Ltd. 